\title{
Forumtheater als Werkzeug szenisch-partizipativer Forschung in der Sozialen Arbeit. Verfahrensweisen, Projektbeispiele und methodische Reflexionen
}

\author{
Michael Wrentschur
}

(C) Der/die Autor(en) 2019

Zusammenfassung Forumtheater ist eine interaktive Theaterform des „Theaters der Unterdrückten“, die in vielerlei Hinsicht zu einem Werkzeug partizipativer Forschung in der Sozialen Arbeit werden kann: Dies betrifft die Einbeziehung von Adressat_innen in die Gestaltungs- und Forschungsprozesse ebenso wie die aktive Beteiligung des Publikums bei Aufführungen und schließlich den Transfer der Ergebnisse in soziale und politische Räume. Ausgehend von grundlegenden methodologischen Überlegungen zu szenischen Forschungsprozessen werden Methodik und Verfahrensweisen des Forumtheaters vorgestellt, mit Projektbeispielen aus Handlungs- und Problemfeldern Sozialer Arbeit illustriert sowie methodisch reflektiert. Diskutiert wird die Frage, welche Ansprüche partizipativer Forschung mit dem Forumtheater realisiert und welche Bezüge zu einer performativen Ethnografie und kritisch-lebensweltorientierten Adressat_innenforschung in der Sozialen Arbeit hergestellt werden können.

Schlüsselwörter Szenisches und partizipatives Forschen · Forumtheater und Soziale Arbeit

\section{Forum Theatre as a tool of drama based, participatory research in Social Work. Methods, examples and methodical reflections}

\footnotetext{
Abstract Forum Theatre is an interactive Theatre technique of the "Theatre of the Oppressed", which can be used as a tool of participatory research in Social Work involving the clients of Social Work into the creation and research process, the active participation of the audience during the performances and the transfer of

M. Wrentschur $(\bowtie)$

Institut für Erziehungs- und Bildungswissenschaft, Universität Graz, Merangasse 70/II, 8010 Graz, Österreich

E-Mail: michael.wrentschur@uni-graz.at
} 
results into social and political spaces. Starting with methodological reflections on drama-based research, methods of the Forum Theatre are presented and illustrated by examples of projects in fields of Social Work. This will be combined with methodical reflections. The question will be discussed, which claims of participatory research can be realized and which references to performative ethnography and critical, living environment orientated user research in Social Work can be made.

Keywords Drama-based and participatory research · Forum Theatre and social work

\section{Einleitung}

Auf welche Weise kann Forumtheater als methodisches Verfahren im Sinne einer szenischen und partizipativen Forschungskonzeption in der Sozialen Arbeit angewendet und in der methodologischen Landschaft verortet werden? Diese Frage hat in meiner eigenen Biografie als Forscher seit meiner Dissertation (Wrentschur 2004) Relevanz, sie wurde in Projekten und Publikationen zu szenisch-partizipativen Forschungsansätzen fortgeführt (Wrentschur 2007, 2008a, 2012) und schließlich in meiner Habilitationsschrift (Wrentschur 2019) stärker methodologisch reflektiert und fundiert. Dabei ist Forumtheater zunächst eine interaktive Aufführungsform des sogenannten Theaters der Unterdrückten $(=\mathrm{TdU})$, bei der das Publikum eingeladen wird, sich am Spielgeschehen zu beteiligen, um vielfältige Handlungs- und Veränderungsideen für einen in szenischen Verläufen dargestellten sozialen Konflikt oder ein Problem zu erproben (Staffler 2009; Boal 2013; Fritz 2013). Die Folgen des Handelns, das besonders auf die Veränderung diskriminierender, ausgrenzender und unterdrückender Interaktionen und Strukturen abzielt, werden in diesem dramatischen Labor unmittelbar sichtbar und erlebbar. Gemeinsam werden Handlungsspielräume untersucht, verbunden mit der Frage, welche strukturellen Veränderungen notwendig sind, um vor allem sozial ausgegrenzten und benachteiligten Gruppen umfassende Möglichkeiten zur gesellschaftlichen (und politischen) Partizipation zu eröffnen. Mit Forumtheater ist aber auch ein partizipativer und szenischer Forschungsprozess in Gruppen gemeint, bei dem sich die Teilnehmenden unter Anwendung szenisch-theatralischer Methoden mit einschränkenden, diskriminierenden oder unterdrückenden Situationen und/oder Strukturen in ihrem Alltagsleben und ihren Lebenswelten auseinandersetzen. Die Theatralisierung und Reflexion von individuellen, subjektiven Erfahrungen führen zu Verdichtungen in Form von Szenen und Bildern. Erkenntnisund Bewusstwerdungsprozesse werden mit der Suche nach Veränderung der persönlichen, sozialen und politischen Wirklichkeit verbunden (vgl. Wrentschur 2012, 2019).

Kommt Forumtheater in Handlungs- und Problemfeldern Sozialer Arbeit zur Anwendung, wird die Einbeziehung von Adressat_innen Sozialer Arbeit in die Gestaltungs- und Forschungsprozesse ebenso wie die aktive Beteiligung des Publikums bei Aufführungen und schließlich der Transfer der Ergebnisse in soziale und politische Räume angestrebt. Damit werden nicht nur Ansprüche partizipativer Forschung realisiert, sondern es zeigen sich Bezüge zu einer performativen Ethnografie und zu einer 
kritischen Adressat_innenforschung. Um das zu verdeutlichen, stelle ich zunächst grundlegende Überlegungen $\mathrm{zu}$ szenischen und performativen Forschungsprozessen an. In weiterer Folge werden Methodik und Verfahrensweisen vorgestellt und mit Projektbeispielen illustriert, bei denen Forumtheater als Werkzeug szenischen Forschens in Problem- und Handlungsfeldern der Sozialen Arbeit zur Anwendung gekommen ist. Dies wird mit methodischen und methodologischen Reflexionen zu Möglichkeiten, Herausforderungen und Grenzen von Forumtheater als Forschungsansatz verbunden.

\section{Vom Theaterspiel(en) als Forschung zur performativen und szenischen Sozialforschung}

Aus der sozialwissenschaftlichen Forschung sind Beispiele bekannt, bei denen Methoden des Theaterspiels zumindest implizit angewendet wurden. Bei den Breaching Experiments der „Ethnomethodologen“ (Garfinkel 1985) wurden alltägliche Regeln und Normen auf irritierende Weise gebrochen, um aufgrund der Reaktionen der Menschen deren alltagssoziologischen Sinn und Zustandekommen besser zu verstehen. Und viele Experimente in der Psychologie wie das Milgram-Experiment (Milgram 1974) können als verdecktes oder unsichtbares Theater bezeichnet werden, bei dem die Versuchspersonen nicht wussten, welche Rollen ihnen im wahrsten Sinn des Wortes vorgespielt wurden, sie agierten in der Als-ob-Realität des Labors, als ob es Realität wäre. Mittlerweile sind vielfältige szenische, performative und theaterpädagogische Verfahren dokumentiert (vgl. Wrentschur 2019, S. 383 ff.), die das Medium ,Theaterspiel“ explizit als Grundlage oder Vertiefung für sozialwissenschaftliche Forschungsprozesse nutzbar machen. So wird festgestellt, dass Theaterschaffende zumeist - wenn auch nicht bewusst - in einem qualitativen Sinn forschen, da es ihnen um ein Verstehen und Interpretieren der sozialen Welt sowie um ein Präsentieren der Ergebnisse der Theaterarbeit in Form von Aufführungen (vgl. Kramer 2007, S. 174) geht. Für Theaterschaffende ist es oft selbstverständliche Praxis, Daten zu sammeln, zu recherchieren und ein Stück zu produzieren, das aus persönlichen Geschichten entwickelt wird. Dabei sieht Kramer in Anlehnung an Denzin den qualitativen, ethnografischen Forscher/die qualitative, ethnografische Forscherin selbst als Performer: ,the world is understood to be performance, culture is a verb, not a noun, fieldwork is a collaborative process - a performance - and knowledge is performative, not informative“ (Denzin 1977, S. 104, zit. nach Kramer 2007, S. 176). Die Rede ist von der performance ethnography, bei der ,leiblich verankerte Interaktionen und deren Bedeutung“ im Zentrum stehen (Winter 2010, Abs. 34). Dabei führen die Ethnograf_innen und zum Teil auch die von ihnen Beforschten die Forschungsergebnisse öffentlich auf, wodurch es zum ,gegenseitigen Austausch und Abgleichen von gelebten Erfahrungen, Emotionen, Perspektiven und Formen des Verstehens“ (ebd.) kommt. Persönliche Geschichten, die Menschen einander erzählen, werden kulturell und gesellschaftlich kontextualisiert, indem sie zu anderen geschriebenen Texten und Diskursen in Bezug gesetzt werden (vgl. Winter 2012, S. 458). Dazu werden in erster Linie vielfältige szenische Verfahren angewendet, um verschiedene Stimmen und Perspektiven der jeweils Betroffenen zu 
präsentieren. Diese Berücksichtigung verschiedener Perspektiven bildet die Voraussetzung für einen Text, ,,der die differenzierten Verschränkungen persönlicher, interpersonaler und politischer Ebenen erfassen kann“. In der Interaktion zwischen den Welten der Forschenden und ,,denen der Erforschten spielt sich der auf Kooperation aufbauende Forschungsprozess ab“ (Winter 2010, Abs. 18).

Auf diese Weise können Zuschauer_innen Verbindungen zu ihren eigenen Erfahrungen herstellen und die Forschungsergebnisse können so für mehr Menschen zugänglich werden als allein für jene, die akademische Textproduktion gewohnt sind und diese studiert haben: „Theatre, I would argue, has the potential of reaching a much wider audience, and also offers a possibility of giving back to a community that one has conducted research in. It further offers multiple ways of communication, through different genres and setting up of spaces, which allow for audience accessibility and even participation, if that is a desired feature" (Kramer 2007, S. 178). Auch für Liamputtong (2007, S. 172ff.) sind Theaterstïcke und Performances Ort und Mittel, um sozialen, politischen und kulturellen Dialog zu verhandeln - Performances können gerade über die Darstellung von Gefühlen die Herzen von Menschen und nicht nur deren Verstand erreichen: „Performance can be a powerful instrument for social reform, for righting some wrongs, and for helping those in need" (Fontana und Frey 2005, S. 714, zit. nach Liamputtong 2007, S. 173). Performative Ansätze in der Ethnografie wie die Autoethnografie oder die performative Ethnografie werden von Winter (2010, Abs. 4) in einem kritischen Verständnis von qualitativer Forschung verortet, gerade dann, wenn sie sozial und politisch im Sinne einer emanzipatorischen Agenda transformativ ,zur Verwirklichung sozialer Gerechtigkeit und einer radikalen Demokratie beitragen“ (ebd.). Durch die szenisch-performative Darstellung von Phänomenen und Problemen können sich alternative Sicht- und Denkweisen entwickeln, was mit dem Ziel verbunden wird, Veränderungen im Alltagsleben von Menschen zu bewirken. Dies geht einher mit kooperativen Formen der Forschung, der Ermächtigung der Beforschten und der Demokratisierung von Wissen und Macht (vgl. Winter 2010, Abs. 16), wie es in der partizipativen Forschung angestrebt wird.

In sozialwissenschaftlichen Forschungskontexten wird zudem von performativer Sozialforschung gesprochen, wie sie etwa in einer Sondernummer vom Forum Qualitative Sozialforschung (9/2008) anhand zahlreicher performativer Verfahren und Konzeptionen vorgestellt und diskutiert wird. Performative Sozialforschung folgt dem wachsenden Interesse am Einbezug von Kunst und dabei vor allem von Drama in den Sozialwissenschaften. Seitz zufolge entstand performative research vor dem Hintergrund einer Kunstpraxis, ,die sich als artistic research gegenüber den Wissenschaften zu behaupten sucht und als practice-led research innerhalb der empirischen Sozialforschung inzwischen sogar einen eigenständigen Status beansprucht" (Seitz 2012, S. 81). Damit wird ,ein eigenes, an künstlerischen Verfahren orientiertes Forschungsparadigma“" befördert (ebd., S. 84). Bei performative research stehen das Handeln und die Alltagspraktiken im Vordergrund, weniger die inneren und äußeren Gründe dafür. Dabei geht es „um ein spezifisches, praktisches Einlassen, um ein umsichtiges Behandeln von sozialer Praxis, von Material, Körper, Raum, ja selbst von Ideen“ (ebd., S. 87). 
Der im vorliegenden Beitrag verwendete Begriff des szenischen Forschens wird in Bezug zu performativen Ansätzen der Sozialforschung gestellt, ist aber stärker an szenisch-theatralischen Vorgangsweisen und Vorgängen im engeren Sinn orientiert (vgl. Wrentschur 2019, S. $381 \mathrm{ff}$.) Dies bedeutet, dass alle Elemente des Theaterspiels dabei eine Rolle spielen (können), wie Raum, Bewegung, Rhythmus, Gestik, Mimik, Körperhaltungen, Emotionen, Sprache, Stimme, Interaktionen, Handlungsabläufe, Rollen und Figuren. Der ästhetische Raum des Theaterspiels ist nach Boal (vgl. 1999, S. 31 ff.) plastisch und gestaltbar, Vergangenes kann gegenwärtig, Fiktives real und Reales fiktiv werden. Darsteller_innen wie Zuschauer_innen sind dabei in einem bestimmten Raum bzw. an einem bestimmten Ort und gleichzeitig in der Welt der jeweiligen Szene(n) anwesend. Und auch die telemikroskopische Dimension des ästhetischen Raumes bekommt für Forschungsprozesse insofern Bedeutung, als Vorgänge und Prozesse vor- und zurückgespult oder auf andere Weise verfremdet werden, damit sich Fremdes im Alltäglichen offenbaren kann (vgl. Boal 1999, S. 27-39; Koch 1997, S. 81-85). Zentral sind jedenfalls die in einem szenischen Raum mitwirkenden Akteur_innen, deren Handlungen, Haltungen und Verhaltensweisen sowie deren Zusammenspiel und Zusammenwirken im theatralischen Raum: „Auf diese Weise näher herangeholt und vergrößert, können menschliche Handlungen besser beobachtet werden“ (Boal 1999, S. 38). Zumeist stehen Inhalte und Themen szenischer Verfahren in Bezug zu Alltags- und Lebenswelterfahrungen, die in szenisch-theatralische Bilder und Handlungsabläufe transformiert und zu einer eigenen ästhetischen wie sozialen Wirklichkeit werden. Dabei gestaltet sich szenisches Forschen ,während der Herstellung und der assoziativen Interpretation notwendigerweise als partizipatorischer Prozess“ (Weitzel 2012b, S. 269). Weitzel bindet szenisches Forschen an die Praxeologie (vgl. Weitzel 2012a, S. $111 \mathrm{ff}$.), deren zentrale Frage es ist, auf welche Weise Menschen handeln (können). Insofern steht eine erkenntnisleitende Handlungsperspektive im Vordergrund und damit eine performative Perspektive auf Soziale Arbeit. Dabei gestaltet sich szenisches Forschen aus Weitzels Sicht,,während der Herstellung und der assoziativen Interpretation notwendigerweise als partizipatorischer Prozess" (Weitzel 2012b, S. 269). Und dies gilt in starkem Maße für Forschungsprozesse mit dem Forumtheater, die ich im folgenden Abschnitt in Bezug zu partizipativen Forschungsansätzen stelle.

\section{Forumtheater im Diskurs partizipativer Forschungsansätze}

In fachlichen Beiträgen und Diskursen zur Aktionsforschung und zur partizipativen Forschung finden sich immer wieder Bezüge zu partizipativer Theaterarbeit wie dem Forumtheater: So verweist Liamputtong (2007, S. 133) darauf, dass Dramamethoden in Projekten der Participatory Action Research (= PAR) häufig eine Rolle spielen. Im aktuellen Handbuch für Action Research berichten Wakefort et al. (2015) über Projekte der PAR, bei denen interaktive und partizipative Theaterformen im Sinne des Forumtheaters zur Anwendung kamen. Guhathakurta (2015) diskutiert ,Theatre in Participatory Action Research“" in Bangladesch sowie in Indien und bezieht sich dabei vor allem auf interaktives Theater im Sinne des TdU. Im Gegensatz zu vielen Entwicklungstheater-Projekten werden der Bevölkerung nicht Lösungen vor- 
gespielt, vielmehr identifizieren die Menschen selbst ihre Probleme, Themen und Anliegen, verbunden mit der Möglichkeit der Transformation (vgl. ebd., S. 101). Streck und Holliday (2015) gehen in Zusammenhang mit der PAR auf lateinamerikanische Wurzeln und Perspektiven ein (vgl. ebd., S. 477 ff.). Sie verweisen u. a. auf das Forumtheater und die Transformation der Zuschauer_innen in die Protagonist_innen der Aktion. Partizipative Aktion und Forschung in ihren verschiedenen Ausprägungen stehen in Lateinamerika in enger Verbindung zu sozialen Bewegungen für soziale Gerechtigkeit. Dies wird auch von Fritz (2013, S. 205 ff.) bestätigt, die sich mit der PAR und der Creación Colectiva in Südamerika auseinandersetzt und Parallelen zum TdU herstellt (vgl. ebd., S. 219): Die PAR wird von impulsgebenden Akteur_innen und der Gemeinschaft durchgeführt, die ihre Realität verändern wollen, wobei die zu bearbeitenden Probleme gemeinsam definiert werden. Praktisch bedeutet dies etwa, dass eine Community eine Einladung an eine/n Forscher_in ausspricht, der/die sich in der Folge mit dem geschichtlichen Kontext des Ortes und der Menschen, mit denen er/sie arbeitet, auseinandersetzt. Die Auswahl der Anliegen und Forschungsmethoden wird gemeinsam entschieden und im Laufe der Forschung gibt es einen Methodentransfer in die einladende Community. Zudem werden alle Ergebnisse in mehreren Schleifen in den größeren Kontext der Gemeinschaft zurückgeführt, damit auf dieser Grundlage neue Schritte zur Verbesserung der Situation geplant werden können (vgl. ebd., S. 260). Aus der Sicht von Fritz (ebd., S. 260 f.) wird dieser Anspruch von der indischen Forumtheatergruppe Jana Sanskriti realisiert: Ausgangspunkte sind Forderungen und Notwendigkeiten der Betroffenen, mit denen gemeinsam über die Vorgangsweise entschieden wird. Gemeinsam wird die Situation analysiert und die Ergebnisse werden in die größere Community zurückgeführt, woraus weitere Vorgehensweisen entwickelt werden. „Der Prozess besteht aus Aktion und Reflexion. Die Beteiligten ,erobern' sich quasi die Situation, sie eignen sich durch den Prozess die Welt als ,Ding für uns “ anstelle eines ,Dings an sich“ an“ (ebd., S. 261). Für Ganguly, den Leiter von Jana Sanskriti, ist Forumtheater in diesem Sinn ein Ort, an dem ,Sozialwissenschaft betrieben wird. Das Resultat ist, dass sich der Prozess der Stückerarbeitung zu einem Prozess entwickelt, in dem Macht in die SchauspielerInnen eingeschrieben wird. Sie fühlen sich der Veränderung ihrer Realität verpflichtet“ (Interview mit Ganguly in Fritz 2011, S. 171).

Im deutschsprachigen Raum widmete sich das universitäre Forschungsprojekt zum „Brüchigen Habitus“ über mehrere Jahre der empirischen Erforschung kooperativer Handlungsmöglichkeiten von Studierenden und Hochschullehrer_innen (vgl. Bülow-Schramm und Gipser 1997, 2007; Gipser 1996, 2007). Dabei wurde in erster Linie das Forumtheater als Methode einer handlungsbezogenen und emanzipatorischen Forschung angewendet, um Machtbeziehungen und Herrschaftsstrukturen im Hochschulalltag zu analysieren und über das dramatische Probehandeln Handlungsalternativen zu finden, die in der Folge in den universitären Alltag übertragen wurden. Ausgehend vom Projekt zum „Brüchigen Habitus“ gibt es von Gipser forschungsmethodologische Überlegungen zum Forumtheater (vgl. Gipser 1996, S. $28 \mathrm{ff} ., 2007$ ). Sie sieht in der „biografischen Selbstreflexion“ - gemeint als Aneignung der eigenen Biografie vor dem Hintergrund gesellschaftlicher Bedingungen durch Aktualisierung und Reflexion eigener vergangener Erfahrungen einen wichtigen Anknüpfungspunkt, vor allem dort, wo es um die Aktivierung des 
Körpergedächtnisses im szenischen Spiel geht. Bezüge werden auch zum sogenannten „,soziologischen Experimentieren“ nach Brecht hergestellt (Gipser 1996, S. 29). Lehr- und Lernprozesse werden in diesem Sinn als ,handlungs- und subjektorientierte Sozialforschungsprozesse" (ebd.) verstanden, biografische Selbstreflexion und soziologisches Experimentieren sind in Hinblick auf Wirklichkeitsveränderung verknüpft. Forumtheater als emanzipatorischer Forschungs- und Bewusstwerdungsprozess basiert auf der Verknüpfung von Forschung und Aktion, Erkennen ist von praktischen und politischen Interessen geleitet und mit der Lebenspraxis verknüpft. Forschungsprozesse mit Mitteln des Forumtheaters speisen sich weniger aus rein akademischen Fragestellungen, sondern aus den Erkenntnisinteressen, Anliegen und Fragen der Mitwirkenden. Gipser (1996, S. 30) sieht darin Möglichkeiten, „Postulate einer emanzipatorischen Handlungsforschung praktisch umzusetzen“, zumal sich deren paradigmatische Elemente wie der dialogische Zugang zur Realität, die Wahrheitsfindung über die kritische Argumentation im Diskurs sowie das zyklische Modell von Datensammlung, Diskussion und Handeln im Feld mit szenischer Forschung gut vereinbaren ließen (vgl. Gipser 1996, S. $29 \mathrm{ff}$.).

Ausgehend von diesen Bezügen des Forumtheaters zu partizipativen und emanzipatorischen Forschungskonzeptionen gehe ich im Folgenden auf Verfahrensweisen und Methoden ein, die bei Forumtheaterprojekten in Handlungs- und Problemfeldern der Sozialen Arbeit Anwendung finden.

\section{Forumtheater in der Sozialen Arbeit: Verfahrensweisen, Methoden und Projektbeispiele}

Grundlage für die Beschreibung von methodischen Verfahrensweisen bilden Forumtheaterprojekte, die in Handlungs- und Problemfeldern Sozialer Arbeit realisiert wurden und in denen sich vielfältige Merkmale und Dimensionen von szenischen und partizipativen Forschungsprozessen zeigen. Die Projekte folgten einem methodischen Grundkonzept, das ich im Folgenden im Überblick vorstelle. ${ }^{1}$ In die Darstellung der Verfahrensweisen werden Beispiele aus Forumtheaterprojekten miteinbezogen (und kursiv hervorgehoben) und methodologische Bezüge hergestellt.

Wesentlich für szenische Forschungsprozesse mit Forumtheater in Handlungsund Problemfeldern der Sozialen Arbeit sind die subjektiven, individuellen Wahrnehmungen und Erfahrungen der Mitwirkenden zu den grundlegenden Themen und Fragen eines Projekts. Zudem können theoretische und fachliche Bezüge (etwa in empirischen Studien zu einer bestimmten Problemlage) in der grundlegenden Frage, die sich ein Projekt stellt, und in der damit verbundenen Gesamtkonzeption eine Rolle spielen und in weiteren Phasen der Stückentwicklung einbezogen werden. In der Regel richten sich Zielsetzungen - wie Unger (2014, S. 53) in Bezug auf partizipative Forschung schreibt - , in erster Linie nach den Relevanzsetzungen der Betroffenen und dem praktischen Handlungsbedarf", wobei sich das Thema und dessen

\footnotetext{
${ }^{1}$ Eingehend beschrieben und diskutiert werden diese Verfahrensweisen in Abschnitt I, II und V meiner bei Beltz Juventa publizierten Habilitationsschrift (Wrentschur 2019). Dabei kommen besonders in Abschnitt V, Fallstudie 3, die Projektmitwirkenden selbst zu Wort.
} 
Fokussierung je nach Relevanz auch ändern können. So bilden thematische Interessen und Anliegen von bestimmten Communitys oder Gruppen, die mit dem Wunsch nach Veränderung verbunden sind, den Rahmen für ein Projekt. Oder es werden Menschen, die bestimmte Erfahrungen (z.B. mit Diskriminierung, Ausgrenzung, finanziellen Notlagen) teilen, eingeladen, an einem szenischen Forschungsprojekt mitzuwirken. Jedenfalls ist wesentlich, dass ein thematisches Interesse der Mitwirkenden besteht, das für die Mitwirkenden in ihren Lebenswelten und in ihrer Praxis bedeutsam ist. Darüber hinaus werden Kooperationen mit sozialen Bewegungen und Organisationen gesucht, die thematische Interessen und Anliegen nach Veränderung teilen. Und aus projektstrategischen Gründen wird überlegt, welche Gruppen und Einrichtungen (Behörden, Politik) eingebunden werden, die für die Umsetzung von Veränderungsanliegen und -vorschlägen relevant sind.

\subsection{Phase 1: Stückentwicklung als emanzipatorischer und partizipativer Gestaltungs-, Recherche- und Erkenntnisprozess}

Szenisches Forschen mit dem Forumtheater wird in Gruppen praktiziert, was in der Regel mit einer hohen Präsenzzeit aller Mitwirkenden verbunden ist. Damit die Teilnahme an den sich oft auch über mehrere Tage, Monate, manchmal sogar Jahre währenden Forumtheaterprojekten möglich ist, müssen die zeitlichen Rahmenbedingungen für die Mitwirkenden lebensweltlich angepasst und mit finanziellen Abgeltungen verbunden werden. So steht am Beginn eines Projekts oft ein mehrtägiger Workshop, bei dem zunächst versucht wird, eine vertrauens- und respektvolle, wertschätzende und kooperativen Atmosphäre in der jeweiligen Projektgruppe herzustellen. Dabei kommen angeleitete Körper-, Bewegungs- und Improvisationsübungen zur Anwendung, über die auch Spiellust und Kreativität angeregt werden. Dies ist eine wesentliche Voraussetzung dafür, dass die Teilnehmer_innen in der Folge Mut fassen, sich den für sie oft belastenden Themen und Problemfeldern zu stellen und diese zu artikulieren. Ähnlich den Prämissen einer kritischen Adressat_innenforschung (Anhorn und Stehr 2012; Hanses 2005; Graßhoff 2013) und der partizipativen Forschung (Unger 2014; Bergold und Thomas 2012) ist bedeutsam, dass sich die Mitwirkenden als Subjekte mit ihren Erfahrungen ernst nehmen und sich individuell und kollektiv Stimme und Ausdruck verschaffen.

Dabei wird auch der Körper als wesentliche Quelle von Wissen und Erkenntnis betrachtet, d.h. körperliche Wahrnehmungen, Bedeutungen, Haltungen und Wertungen eines Themas werden in den Forschungsprozess miteinbezogen. Das kann sich auf das Anregen der Körpererinnerung, auf das Bewusstmachen von routinisierten, einverleibten Körperhaltungen oder auf das Aufspüren von gesellschaftlich mitproduzierten Bewegungsmustern sowie auf die nonverbalen und symbolhaften Ausdrucksmöglichkeiten und Dimensionen eines Themas beziehen. So werden die subjektiven Erfahrungen und Perspektiven der Mitwirkenden besonders über nonverbale, körperbezogene Ausdrucksformen wie den vielfältigen Bildertheater-Techniken artikuliert, bei denen soziale Situationen, Erfahrungen, Geschichten und Probleme der Teilnehmer_innen mithilfe von (eingefrorenen) Körperhaltungen, Gestik und Mimik dargestellt werden (vgl. Wrentschur 2008a, S. 103). „Image and imagination thus become an interplay of structure and de-structure, the image providing a form 
of closure to play with; the imagination providing a way of opening up the form to possibility“ (Linds und Vettraino 2008, Abs. 7). Diese methodische Vorgangsweise unterstützt die Teilnehmenden dabei, Momente und Erfahrungen aus ihrem Alltag, ihrer Lebenswelt zum Ausdruck zu bringen, ästhetisch zu verdichten, zu verfremden, zu betrachten und kreativ zu bearbeiten. Oft erkennen Projektmitwirkende Aspekte ihrer eigenen lebensweltlichen Erfahrungen in theatralischen Bildern, Szenen und Geschichten, die von anderen Teilnehmer_innen herrühren, wieder. Zudem werden die symbolisch verdichteten Bilder und deren gemeinsame Analyse zu einem bedeutsamen Impuls für Austausch und Diskussionen unter den Mitwirkenden über die gezeigten und erlebten Situationen und Problemlagen.

Im Zuge des Projekts „Legislatives Theater mit wohnungslosen Menschen“ (Wrentschur 2008a) wurde ausgehend von bewusst provokant gestellten Fragen wie „Was empört euch in Bezug auf Wohnungslosigkeit? Was findet ihr ungerecht? Was müsste am dringendsten verändert werden? “ die Gruppe dazu angeregt, im Sinne des Bildertheaters „lebende Skulpturen“ zu entwickeln und darzustellen. Der Wechsel zwischen dem szenischen Gestalten, dem Beobachten, dem Reflektieren und Diskutieren war eine entscheidende Triebkraft dafür, dass die oftmals mit Gefühlen von Ohnmacht, Scham und Hilflosigkeit verbundenen Erfahrungen von Wohnungslosigkeit artikuliert werden konnten. Dabei wurden besonders Situationen zum Ausdruck gebracht, in denen sich wohnungslose Menschen mit Ausgrenzung, Diskriminierung oder Respektlosigkeit konfrontiert sahen, oder in denen es als Herausforderung erlebt wurde, sich im System der angebotenen (und verwehrten) sozialen Dienste und Unterstützungsleistungen zurechtzufinden.

Die Mitwirkenden bringen ihre persönliche Ansicht in die gemeinsame Auseinandersetzung ein und geben Impulse für Verständigungs-, Interpretations- und Assoziationsprozesse. Dokumentiert wird dies schriftlich in Form von Protokollen mit Beschreibungen und Assoziationen zu den einzelnen Bildern, oft auch in Stichworten auf Plakaten in Kombination mit Fotos. Dies gilt vor allem als Erinnerungsstütze und in weiterer Folge als Grundlage für weitere Entscheidungen der thematischen Vertiefung und szenischen Gestaltung. Im Sinne von „Daten“ sind jedoch die entwickelten Bilder und Szenen vorrangig. Davon ausgehend wird verstärkt nach den verbindenden, gemeinsamen Problem- und Konfliktlagen gesucht, es wird auf intersubjektive Themen und auf die damit verbundenen (gemeinsamen) Anliegen nach Veränderung fokussiert. Dafür bieten sich unterschiedliche methodische Möglichkeiten an, wie etwa das sogenannte Bild der Bilder, in dem Aspekte und Dimensionen bereits zum Ausdruck gebrachter theatralischer Bilder verbunden und analysiert werden (vgl. Boal 1999, S. 74 ff.) oder auch das Magnetische Bild (vgl. Diamond 2013, S. $152 \mathrm{ff}$.), bei dem Projektmitwirkende übergreifende Themen ihrer gemeinsamen Erfahrungen zum Ausdruck bringen. Zur Relevanzsetzung und Priorisierung übergreifender Themen können - über die theatralische Arbeit und den Erfahrungsraum der Gruppe hinaus - Recherchen in Form von (aktivierenden) Befragungen, aber auch andere Recherche- und Forschungsmethoden wie Sozialraumbegehungen oder Medienanalysen Anwendung finden.

So wurden beim Projekt „Legislatives Theater mit wohnungslosen Menschen“ Rückkopplungsschleifen zur Community wohnungsloser Menschen in Graz gebildet (vgl. Wrentschur 2008a, S. 102f.). Die Gruppenmitglieder betätigten sich als Sozi- 
alforscher_innen und führten kurze Fragebogeninterviews mit wohnungslosen Menschen durch, um zu erfahren, welche Probleme und Anliegen von den wohnungslosen Menschen als besonders dringend angesehen werden. Als selbst Betroffene konnten die Gruppenmitglieder das Vertrauen ihrer,Kolleg_innen' gewinnen, die Vorarbeiten innerhalb der Projektgruppe kamen ihnen dabei zugute. Die Ergebnisse dieser Befragung bestärkten die Gruppe in ihrer Themenwahl, sie wurden zu einer wertvollen Stütze und Rückbindung für die weitere Entwicklung der Szenen und Bilder. Die Themen, Problemlagen und Anliegen wurden schließlich nach ihrer Dringlichkeit gereiht, wobei Schwierigkeiten und Diskriminierungen bei der Arbeits- und Wohnungssuche, bürokratische Barrieren und mangelnder Zugang zu relevanten Informationen (das „Herumgeschickt-Werden“) sowie die Verschuldensdynamik zu den wichtigsten zählten.

Schließlich werden aus dem gemeinsam entwickelten, szenischen Rohmaterial eine oder mehrere ästhetisch-theatralische Szenen oder Bilder gestaltet. Dabei soll deutlich werden, welches Problem im Fokus steht und welche Fragen in den jeweiligen Szenen bzw. mit dem Stück aufgeworfen werden. Parallel dazu spielen ästhetische und szenische (Verdichtungs-)Prozesse eine große Rolle. Methodisch kommen dabei zumeist Formen der Improvisation mit anschließenden Reflexionen sowie spezielle Probetechniken zur Fokussierung und Gestaltung der szenischen Abläufe zur Anwendung. Folgende Fragen stehen im Zentrum: Was braucht es an Handlungen, an Gefühlen und räumlichen Arrangements, an Gesten und Haltungen, damit die Szene stimmiger und realer wird, damit die in den Situationen wirkenden Macht- und Statusbeziehungen deutlich und den von der Gruppe intendierten Problemen und Anliegen gerecht werden? Sind die sozialen Rollen und deren Habitus bzw. Haltungen entsprechend dargestellt? Sind die Szenen entsprechend vielschichtig und komplex? In welcher Weise berühren die Szenen die gemeinsamen Erfahrungen und/ oder Anliegen der Mitwirkenden?

Neben dem ästhetischen Entwicklungs- und Gestaltungsprozess ist in dieser Phase vor allem die Rollenarbeit sehr bedeutsam, um die Darsteller_innen gut auf die interaktive Forumphase vorzubereiten, bei der diese aus dem Wissen und Habitus einer Rolle heraus auf Veränderungsideen der Zuschauer_innen reagieren. Unterstützt wird dies mit Recherchen zu Fakten, Gesetzen, Abläufen und Handlungsspielräumen der beteiligten Rollen.

Beim Forumtheaterprojekt „Kein Kies zum Kurven Kratzen“ (Wrentschur 2019, S. 787) führte ein Darsteller, der selbst mit finanziellen Notlagen zu kämpfen hatte, Interviews mit Gerichtsvollzieher_innen durch, um für die Darstellung seiner Rolle im Stück Informationen über gesetzliche Vorgaben und Handlungsspielräume zu bekommen. Und beim gleichen Projekt setzte sich eine Akteurin, die das AMS viele Jahre lang aus der Perspektive einer arbeitslosen Person erlebt hatte und im Forumtheaterstïck eine AMS-Beraterin spielte, mit den Perspektiven und Handlungsräumen von diesen auseinander. Hier fand schon bei der Recherche ein Rollen- und Statuswechsel statt: Die Rolle als Darstellerin und Forscherin eröffnete nicht nur Zugänge zu Informationen, sondern erlaubte Gespräche auf Augenhöhe.

Ein weiteres methodisches Element in dieser Phase bildet eine Form der dialogischen Rückkopplung und Validierung, indem Menschen, die aufgrund ihrer Betroffenheit eine persönliche oder eine professionelle Expertise zu den erarbeiteten 
Themen aufweisen, zu offenen Proben eingeladen werden. Dabei werden szenische Entwürfe präsentiert, um gemeinsam zu reflektieren und zu diskutieren, inwieweit sie den Erfahrungen und Realitäten der Eingeladenen entsprechen. Im Sinne eines Wissens- und Informationstransfers werden zudem szenische Improvisationen zum Kontext und Inhalt der Szene durchgeführt, an denen die eingeladenen Expert_innen unmittelbar mitwirken. Diese offenen Proben dienen dem Austausch relevanter Informationen zum Thema, die einen wichtigen Hintergrund für die Szenen bilden. Dies kann zu weiteren Recherchen zu fachlichen, gesetzlichen oder politischen Hintergründen anregen, die für die jeweiligen Szenen relevant sind.

Dieser szenische Forschungsprozess führt in ein von Text und Ablauf her fixiertes Forumtheaterstück, das ästhetisch gestaltet und verdichtet Erkenntnisse und Phänomene des bisherigen Gestaltungs- und Reflexionsprozesses enthält. Die Mitwirkenden eines Forumtheaterprojekts sind in allen bisherigen Phasen des szenischen Forschens mit dem Forumtheater aktive Subjekte und Akteur_innen der Forschung, die ihre individuellen Erfahrungen und Sichtweisen zu gemeinsam gestalteten, kollektiven theatralischen Bildern und Szenen verbinden. In den szenischen Verläufen werden individuelle, alltägliche biografische Verläufe und Handlungsverläufe in ihrem dialektischen Wechsel- und Spannungsverhältnis zu gesellschaftlichen und strukturellen Rahmenbedingungen und Kontexten dargestellt. Diese szenisch verdichteten Erkenntnisse werden in der Folge einem Publikum vermittelt, verbunden mit einer oder mehreren Frage(n), die bei den interaktiven Aufführungen bearbeitet werden. Dies setzt gemeinsame Klärungen darüber voraus, was die Projektmitwirkenden öffentlich zeigen wollen - Text und Handlungsablauf bedürfen der Zustimmung aller.

\subsection{Phase 2: Interaktive Aufführungen als kollektive und partizipative Forschungsforen}

Mit den interaktiven Aufführungen wird die in offenen Proben realisierte dialogische Rückkopplung intensiviert, indem vor allem betroffene Communitys, einschlägige Organisationen und Initiativen sowie gesellschaftliche Öffentlichkeiten angesprochen und einbezogen werden. Über eine Aufführungsserie hinweg kann sich auf diesem Weg in umfassenderer Weise herauskristallisieren, inwieweit die dargestellten Szenen als Ergebnisse der szenischen Forschungsprozesse kontextualisierbar und über die Erfahrungen und Erkenntnisse der Projektmitwirkenden hinaus generalisierbar sind, gerade auch, weil es dabei nicht um eine Reproduktion sozialer Wirklichkeit geht, sondern um deren szenische und ästhetische Verdichtung. Forumtheateraufführungen erweisen sich als eine Form einer intervenierenden und kommunikativen Feldforschung, bei der regionale Eindrücke, Befunde und Erkenntnisse zur Wahrnehmung und zum Umgang mit den gezeigten sozialen Problemen aufgenommen und analysiert werden können. Dieser Erkenntnisprozess kann in der Folge unter Anwendung anderer Methoden (regional) vertieft werden (vgl. Wrentschur 2008b, S. 717 f.).

Szenisches Forschen mit dem Forumtheater ist in der interaktiven Forumphase von einem spielerisch-experimentellen Charakter geprägt. In dieser Phase wird gemeinsam mit dem Publikum nach Ideen zur Veränderung bzw. zu Lösungsansätzen für die gezeigten Probleme und Konflikte gesucht, die auch hinsichtlich ihrer Über- 
tragbarkeit reflektiert werden: Szenen werden unterbrochen und in neuen Varianten gespielt; es wird zwischen der unmittelbaren Beteiligung im Spiel, der Beobachtung und der Reflexion gewechselt. Die Forumphase gleicht dem angesprochenen soziologischen Experimentieren mit Haltungen, Verhaltensweisen und Handlungen (vgl. Koch 1988, S. 45), indem versucht wird, dem Geschehen auf der Bühne eine Wendung zu geben, während die Darsteller_innen aus der habituellen Perspektive der jeweiligen Rolle darauf reagieren. Dadurch werden mögliche Folgen des Handelns unmittelbar sichtbar und erlebbar gemacht, genauso wie jene habituellen, soziokulturellen und strukturellen Barrieren bzw. Grenzen, auf die individuelles Handeln stoßen kann (Wrentschur 2019, S. 416f.).

In zahlreichen interaktiven Forumtheateraufführungen wurde beim bereits erwähnten Projekt „Legislatives Theater mit wohnungslosen Menschen“ (Wrentschur 2008a) das Publikum, das sich aus wohnungslosen Menschen, in sozialen Einrichtungen Tätigen und Interessierten zusammensetzte, dazu eingeladen, sich im Sinne des Forumtheaters am Spielgeschehen zu beteiligen, die Rolle des oder der Wohnungslosen zu ersetzen und Veränderungsideen auszuprobieren. Das Erkenntnisinteresse galt vor allem der Frage, wie es möglich ist, aus dem nach unten führenden Kreislauf auszusteigen und auf diskriminierende und ausgrenzende Praktiken zu reagieren. Die „Einstiege“ in die Forumtheaterszenen loteten Handlungsspielräume der Rollen in den jeweiligen Szenen aus und ließen erkennen, welche institutionellen oder gesetzlichen Veränderungen notwendig wären, um die Reintegration und Partizipation von wohnungslosen Menschen zu erleichtern. Als große Hürde stellten sich dabei die eklatanten Macht- und Statusunterschiede heraus, mit denen wohnungslose Menschen konfrontiert sind. Vor dem Hintergrund des zumeist geringen sozialen, ökonomischen, kulturellen und symbolischen Kapitals, auf das wohnungslose Menschen zurückgreifen können, wurde die Suche nach Möglichkeiten angeregt, den dargestellten Machtungleichgewichten zumindest insofern zu begegnen, als wohnungslose Menschen in den für sie existenziell bedeutsamen Situationen als Dialogpartner_innen ernst genommen werden. Dieser Prozess machte beschränkende oder beherrschende habituelle Praktiken bewusst, denen wohnungslose Menschen selbst unterliegen bzw. mit denen sie konfrontiert sind. Es zeigte sich das für die individuelle Handlungsebene interessante und zugleich widersprüchliche Ergebnis, dass es durchaus Wege gab, um aus der Sicht wohnungsloser Menschen schwierige Situationen zu bewältigen oder zu wichtigen Auskünften und Unterstützungsleistungen zu gelangen. Dafür schienen etwa selbstbewusstes oder hartnäckiges Auftreten, Freundlichkeit und Humor, das Deutlichmachen von Notsituationen, das Aushandeln von Lösungen sowie das Umdeuten von Situationen notwendig. Die meisten dieser Eigenschaften und Verhaltensweisen stehen aber im Widerspruch dazu, dass akut wohnungslose Menschen in der Regel zumeist über Scham- und Versagensgefühle und über wenig Selbstwert und Selbstbewusstsein verfügen, was oftmals destruktive oder aggressive Verhaltensweisen befördert. Insofern bestand eine wesentliche Erkenntnis darin, dass die Forderung nach Veränderung der individuellen Haltungen der Betroffenen zu kurz greift, wenn soziale Unterstützungssysteme und Behörden ihrerseits Barrieren aufbauen und damit Ausgrenzungspraktiken vollziehen, welche die rechtlichen Ansprüche wohnungsloser Menschen auf Information und Hilfestellungen konterkarieren (vgl. Wrentschur 2008a, S. 106). 
Der Erprobung von Handlungsalternativen bei den Einstiegen folgt eine gemeinsame Reflexion, indem Einsteiger_innen, Zuschauer_innen und manchmal auch Darsteller_innen ihre Wahrnehmungen und Ansichten artikulieren. Die spielerischexperimentelle Auseinandersetzung mit theatralisch verdichteten Lebens- und Alltagswelten trägt zu einem besseren Verstehen der Handlungen der einzelnen Akteur_innen, der gezeigten sozialen Probleme und ihrer Auswirkungen bei: Durch Einstiege können bislang verborgene, auch tabuisierte oder zunächst unbewusste Facetten eines Themas zutage treten und dessen Komplexität und Vielschichtigkeit deutlich werden. Entlang der zentralen Frage, ob eher individuelle, handlungsbezogene und/oder eben eher strukturbezogene, politische Herangehensweisen notwendig sind, um Veränderungen $\mathrm{zu}$ initiieren und $\mathrm{zu}$ realisieren, verstehen sich Forumtheateraufführungen als handlungs- und dialogorientierte Forschungsprozesse. Wie bei Ansätzen der performativen Ethnografie schaffen die Forumtheateraufführungen einen gemeinsamen Erfahrungsraum, in dem zwischen Darsteller_innen und Publikum Erlebnisse, Emotionen und Verständnisse geweckt, entfaltet und geteilt werden können. Das Publikum wird zum Ko-Produzenten der Forschung und der Geschichten, die weitergeschrieben, umgedeutet, neu geschrieben werden. Damit wird ein Raum für Reflexion, Diskussion und Dialog eröffnet, der zu einem Wandel von Einstellungen und Perspektiven führen kann, indem übernommene Bedeutungen und normative Traditionen über Performance aufgebrochen, in Bewegung gebracht, hegemoniale Strukturen infrage gestellt und demokratische, partizipatorische sowie emanzipatorische Perspektiven unterstützt werden. „Performance wird auf diese Weise zu einem ,umfassenden und integrierenden Konzept, um kulturelle und soziale Prozesse verstehen und verändern zu können“ (Winter 2010, Abs. 36).

Bei Projekten, die stärker auf die Veränderung politischer Rahmenbedingungen abzielen, wird das Publikum auch dazu eingeladen, aus den Erfahrungen der interaktiven Aufführungsprozesse heraus strukturell-politische Anliegen und Vorschläge $\mathrm{zu}$ formulieren, die sich aus der Auseinandersetzung mit dem Stück ergeben und die Suche nach eher individuellen Handlungsmöglichkeiten ergänzen. Alle Einstiege, Diskussionsbeiträge und Vorschläge, die im Verlauf einer Aufführung entstehen, werden jedenfalls schriftlich, manches Mal auch audiovisuell über Filmmitschnitte dokumentiert.

\subsection{Phase 3: Auswertung und Transfer}

Schon während der Forumtheateraufführungen werden in Zusammenhang mit den Einstiegen des Publikums gemeinsam Handlungsmöglichkeiten reflektiert. Dadurch kann es zu vielfältigen Erkenntnissen kommen, die mit dem unmittelbaren und oft auch körperlichen Erleben der Aufführungen in Zusammenhang stehen. Diese Erkenntnisarbeit setzt sich im Projektteam aber über die Aufführungen hinaus fort und regt zur Frage an, wie die gewonnenen Erkenntnisse in die jeweiligen Lebensrealitäten übertragen werden können bzw. welche Lösungsvorschläge und Handlungsansätze auf persönlicher, gesellschaftlicher und politischer Ebene damit verbunden sind. Grundlage dafür sind die inhaltsanalytische Auswertung und Strukturierung der in den Protokollen dokumentierten Einstiege, Lösungsvorschläge und Diskussi- 
onsbeiträge des Publikums, die in der Gruppe der Projektmitwirkenden hinsichtlich zweier grundlegender Perspektiven reflektiert und diskutiert werden:

Zum einen wird untersucht, ob sich in den über die Einstiege des Publikums zum Ausdruck gebrachten Lösungsideen und Handlungsalternativen Ansätze zeigen, die für die Mitwirkenden am Projekt als sinnvoll, bedeutsam und in ihren Lebensrealitäten als realisierbar erscheinen. Dazu werden in der Gruppe der Projektmitwirkenden die Einstiege und ihre Wirkungen gemeinsam besprochen und reflektiert, und es wird überlegt, wie Haltungen und Handlungsweisen, die im szenischen Spiel als wirksam und stimmig erlebt werden, in der Folge im Alltag bzw. in der gesellschaftlichen Praxis auf die Probe gestellt werden. Handlungsmöglichkeiten beziehen sich dabei zumeist auf Situationen und Dynamiken von Ausgrenzung, Unterdrückung und Ohnmacht, sie können persönliche, soziale, berufliche und politische Kontexte betreffen. Über individuelle Handlungsansätze hinaus können zudem gemeinsame, kollektive Handlungsformen angedacht und realisiert werden.

Zum anderen geht es um die Frage, ob in den Einstiegen und formulierten Lösungsvorschlägen generelle, verbindende Vorschläge und Lösungsansätze gefunden werden können und daraus ein gesellschaftlicher, politischer oder behördlicher Handlungs- bzw. Veränderungsbedarf artikuliert werden kann. Auf dieser Grundlage werden Vorschläge, Lösungsansätze, Anliegen und Forderungen von der Gruppe der Projektmitwirkenden formuliert, die zur Verbesserung bzw. Veränderung einer bestimmten sozialen oder politischen Problematik beitragen können. Diese Vorschläge, Lösungsideen und Forderungen können anschließend mit weiteren betroffenen und professionellen Expert_innen hinsichtlich ihrer Sinnhaftigkeit, ihres Beitrags zur Lösung der thematisierten Probleme reflektiert und diskutiert werden. Dabei ist die Frage wesentlich, ob die Vorschläge und Lösungsansätze Betroffene in einer ähnlichen Lage unterstützen können, ihre Situation besser zu bewältigen. In einem weiteren Schritt können die Vorschläge und Ideen gemeinsam mit professionellen Expert_innen dahingehend überprüft werden, welche politischen, behördlichen oder rechtlichen Kontexte davon berührt werden. Nach einer nochmaligen Überarbeitung der Vorschläge und Texte - wieder unter Miteinbeziehung von betroffenen und fachlichen Expert_innen - werden diese für den Transfer an unterschiedliche Entscheidungsträger_innen aufbereitet. Diese partizipative Produktion eines Berichts mit Vorschlägen und Forderungen ist Ergebnis eines kollektiven Reflexions- und Klärungsprozesses, der die Erfahrungen und Sichtweisen von Menschen ernst nimmt, die eine Problematik aus der eigenen Lebenswelt kennen und die sich in einem länger währenden Forschungs- und Bewusstseinsprozess über ihre Anliegen und Veränderungsvorschläge klargeworden sind.

Unter Zuhilfenahme der jeweiligen Forumtheaterstücke kann in der Folge in unterschiedliche behördliche und politische Kontexte interveniert werden, um die Vorschläge, Anliegen und Lösungsideen mit Entscheidungs- und Verantwortungsträger_innen in Bezirken, Kommunen, Ländern oder im Parlament zu diskutieren (vgl. etwa Wrentschur 2008a, 2008b, 2010, 2011, 2012; Wrentschur und Moser 2014). Die Forumtheaterstücke haben dabei nicht nur die Funktion, ästhetisch verdichtete Ergebnisse des szenischen Forschungsprozesses an Entscheidungs- und Verantwortungsträger_innen zu vermitteln, sondern dadurch die bei den bisherigen Aufführungs- und Reflexionsprozessen entwickelten Lösungs- und Veränderungsideen ver- 
ständlich und nachvollziehbar zu machen. Dabei spielt besonders die Frage eine Rolle, wie die Vorschläge und Anliegen von den jeweiligen Verantwortungsträger_innen umgesetzt werden können.

Das Projekt „ZusammenSpiel“ konzentrierte sich auf das Zusammenleben in einem Grazer Park und versuchte, Konflikte zwischen Benutzer_innengruppen in Szene zu setzen und partizipativ zu verhandeln (vgl. Wrentschur 2019, S. 615ff.). Bei Interviews mit Expert_innen, Sozialraumanalysen und bei offenen Proben im Park zeigte sich, dass der Erholungswert im Fröbelpark im Konflikt mit dem Fröbelpark als Spielplatz steht. Da es in diesem Stadtteil sonst keine öffentlichen Spielplätze gibt, wird der Raum für die spielenden Kinder enger, die aufgrund der Wohnsituation oft viel Zeit im Freien verbringen (müssen). Diese räumliche Enge sorgt für Konflikte zwischen Fußball spielenden Burschen und Mädchen (die Burschen sind mit ihrem Fußballspiel sehr platz- und raumgreifend, die Mädchen fühlen sich davon sehr eingeschränkt und gestört), für Konflikte mit Anrainer_innen (Lärm, Bälle, die gegen Autos, Zäune etc. fliegen), aber auch mit Eltern, die mit ihren Kleinkindern im Park sind (die Bälle fliegen oft gefährlich knapp an Kinderköpfen vorbei, manchmal auch darauf ...). Die Darsteller_innen des Forumtheaters waren ortskundige Jugendliche, gemeinsam mit dem anwesenden Publikum (Parkbenutzer_innen, Eltern, Geschwister, angrenzende Bewohner_innen) wurde im interaktiven Forumtheaterprozess nach Lösungsideen und neuen Handlungsmöglichkeiten gesucht. Unmittelbar im Anschluss an die zweite Aufführung fand eine Diskussion statt, an der neben den jugendlichen Projektmitwirkenden, Vertreter_innen der kommunalen Politik sowie von umliegenden Schulen und Jugendeinrichtungen teilnahmen. Allen Beteiligten war klar, dass mehr freie, bespielbare Flächen im öffentlichen Raum benötigt werden, was bei der Stadtplanung und den Bebauungsplänen aber nicht berücksichtigt wird. Als ein Ergebnis der Diskussion nach der Aufführung wurde eine schnelle, kurzfristige Lösung für die Sommerferien ermöglicht: Im Sinne der Mehrfachnutzung vorhandener Sportplätze wurde der Sportplatz einer angrenzenden Schule wochentags von 13-18 Uhr geöffnet und entsprechend betreut. Ein Konzept, das schon lange in den Schubladen gelegen ist, wurde als Folge des partizipativen Theaterprozesses und des Erlebens der Forumtheateraufführung Wirklichkeit und in der Folge zu einem Modellfall für die gesamte Stadt Graz.

\section{Methodische Reflexionen und Ausblicke}

Forumtheater lässt sich in unterschiedlicher Akzentuierung als Forschungsprozess gestalten, wobei emanzipatorische und partizipative Gestaltungs-, Recherche- und Erkenntnisprozesse bei der Stückentwicklung, die interaktiven Aufführungen als kollektive und partizipative Forschungsforen und schließlich die Auswertung und der Transfer ins Soziale und Politische als wesentliche Phasen beschrieben werden können. Neben erfahrungs- und handlungsbezogenen Erkenntnissen im szenischen Forschungsprozess sind Forumtheaterszenen und -stücke, die in ästhetisch verdichteter Weise Erkenntnisse über das Zusammenspiel von sozialen Handlungen, Dynamiken und Strukturen beinhalten, ein zentrales Ergebnis dieses Prozesses. Die Theatralisierung von Erfahrungen aus dem sozialen Alltag und von sozialer Wirklichkeit 
generiert Wissen und Erkenntnisse, ebenso wie die gemeinsame Stückentwicklung, die damit verbundene Rollenarbeit und die interaktiven Aufführungen. Zudem werden Berichte zum Projektprozess und zu inhaltlichen Ergebnissen auf Basis der dokumentierten, ausgewerteten und rückgekoppelten Lösungs- und Veränderungsvorschläge verfasst.

Ähnlich der partizipativen Forschung und der ethnografischen Forschung im Kontext einer kritischen qualitativen Forschung versteht sich szenisches Forschen mit dem Forumtheater als Auslöser für sozialen Wandel sowie als eine in soziale und politische Räume eingreifende Forschungsstrategie. Die Reflexions- und Erkenntnisprozesse beim Forumtheater sind vom Wunsch und von der Suche nach Veränderungsmöglichkeiten hinsichtlich der Überwindung von Unterdrückung, Ausgrenzung und Diskriminierung in Richtung von mehr sozialer Gerechtigkeit, Teilhabe, Partizipation und Empowerment geleitet. Insofern ist es ein Ziel, in starkem Maße Veränderungswissen zu produzieren, das zu den lebensweltlichen Bedingungen und Lebenslagen der Projektmitwirkenden rückgebunden ist. So stehen die Forschungsprozesse in starkem Zusammenhang mit alltäglichen Erfahrungen in der Lebenswelt der Teilnehmer_innen, die mit theatralen Werkzeugen artikuliert, verdichtet, verfremdet und transformiert werden, um damit Problemlagen und generative Themen spezifischer Gruppen und Communitys bewusst zu machen. Diese gemeinsame Untersuchung lebensweltlicher Verhältnisse macht es möglich, Konfliktverhältnisse im Alltag zu rekonstruieren und diese ,in ihrer Dialektik als Kritik und Handeln, Zwang und Freiheit wie auch Unterdrückung und Emanzipation zu beschreiben“ (Schimpf 2012, S. 258), was sich nicht nur mit den Zielen der kritischen Adressat_innenforschung, sondern auch mit der performativen Ethnografie und Ansätzen der partizipativen Forschung verbinden lässt. Dabei ist wesentlich, welchen Nutzen und welchen Gebrauchswert die Mitwirkenden aus den Erkenntnissen für ihr Handeln und ihre Handlungsspielräume schöpfen - im Sinne der Erweiterung des Wissens und der gemeinsamen Handlungsfähigkeit aller Beteiligten. Zentral ist das Kennenlernen neuer Perspektiven und Handlungsmöglichkeiten auf persönlicher, sozialer und politischer Ebene, gerade auch dort, wo es um soziale Probleme und Konflikte, um Prozesse von Ausgrenzung, Macht und Unterdrückung geht.

Der szenische Forschungsprozess ist maßgeblich von Menschen getragen, deren Stimmen und Perspektiven nur selten einbezogen werden ,und die selbst kaum Möglichkeiten haben, ihre Interessen begründet einzubringen und durchzusetzen" (Bergold und Thomas 2012, S. 19), die aber von den Umständen, unter denen sie leben bzw. denen sie ausgesetzt sind, am meisten betroffen sind. Forumtheater eröffnet gerade gesellschaftlich ausgegrenzten oder marginalisierten Gruppen die Möglichkeiten zur Artikulation ihrer Stimmen und Sichtweisen. In der jeweiligen (sozialen) Standpunktperspektive kann ein Wissen elaboriert und artikuliert werden, das die dominanten Diskurse und Praktiken umgeht, indem die Welt aus der Perspektive der Betroffenen gezeigt wird und Strukturen von Unterdrückung dargestellt werden. Über vielschichtige und vielstimmige Aufführungstexte werden soziale Probleme und deren Komplexität in der Verbindung von Alltagshandeln und gesellschaftlichpolitischer Struktur in einer Weise zum Ausdruck gebracht, die viele Menschen weit über akademische Kontexte hinaus erreichen und beteiligen kann. Die Emotionalität und Vielschichtigkeit in den dargestellten Forumtheaterszenen regen dazu an, 
Verbindungen zu eigenen lebensweltlichen Erfahrungen herzustellen, die Wissensund Erkenntnisproduktion realisiert sich nicht nur über Texte, sondern auch über Handlungen, Haltungen, Gestik und emotionales Erleben. Eine derart verstandene aufführungsorientierte Sozialwissenschaft wird durch eine kritische soziologische Imagination geprägt, die eine radikale Demokratisierung der Lebensverhältnisse anstrebt. „Dabei ist es Aufgabe einer kritisch orientierten Sozialwissenschaft, Erfahrung, Politik, Performativität und Ermächtigung miteinander zu verknüpfen" (Winter 2010, Abs. 40).

Dies alles erweist sich insgesamt als voraussetzungsvoll und ist mit speziellen Herausforderungen und Spannungsfeldern verbunden. So liegt in der Stärke dieses Ansatzes zugleich eine Schwierigkeit: Erst die notwendige hohe Präsenzzeit der Projektmitwirkenden eröffnet vielfältige Reflexions- und Erkenntnismöglichkeiten und macht die Entwicklung von qualitätsvollen Forumtheaterstücken möglich. Die mit der Teilnahme einhergehende Involviertheit und intensive Auseinandersetzung mit belastenden, oft auch schmerzhaften Themen kann als sehr herausfordernd und anstrengend erlebt werden. Umso wichtiger sind transparente und unterstützende Rahmenbedingungen, eine auf Wertschätzung und Respekt basierende Zusammenarbeit und die mit der Theaterarbeit verbundenen Räume für Kreativität, Ausdruck und gemeinsames Handeln.

Herausfordernd ist zudem, dass inhaltlich und ästhetisch überzeugende Forumtheaterstücke notwendige, aber nicht hinreichende Voraussetzung für deren soziale und politische Wirkung sind. Vielmehr ist bei den jeweiligen Projekten viel an Vernetzungs- und Öffentlichkeitsarbeit zu leisten, die von Projektbeginn an mitgedacht werden muss. Nur so können über die Aufführungen viele Menschen aus gesellschaftlichen, professionellen, behördlichen oder politischen Kontexten angesprochen, erreicht und am Dialog über Lösungsansätze beteiligt werden. So waren beim Projekt „ZusammenSpiel“ die Recherchen und Gespräche mit relevanten Akteur_innen im Stadtteil nicht nur hinsichtlich deren Sichtweisen und Einschätzungen bedeutsam, sondern auch, um diese in ihrer Kooperations- und Veränderungsbereitschaft anzusprechen. Abgesehen davon müssen in Bezug auf die Veränderungsanliegen und -vorschläge wiederholt Rückkopplungen zum Projektteam, zu relevanten Communitys, Initiativen und Menschen mit einschlägiger Expertise erfolgen. Dies alles ist eine Grundlage für politische Folgehandlungen, die aber außerhalb der Gestaltungsmöglichkeiten und des Machtbereichs eines Projekts liegen. So hat sich im Zuge von Forumtheaterprojekten immer wieder herausgestellt, dass die „Stimmen der Adressat_innen“"zwar beim unmittelbaren Erleben der Aufführungen Gehör finden und bei politischen Entscheidungsträger_innen Verständnis und Umsetzungsbereitschaft für die Veränderungsvorschläge geweckt wird, dass diese aber aufgrund der Dynamiken innerhalb der jeweiligen politischen Gremien nicht realisiert werden (vgl. Wrentschur 2014).

Zur weiterführenden und vertiefenden methodischen und methodologischen Entwicklung wird die Realisierung weiterer Projekte, deren Reflexion in Publikationen und innerhalb und außerhalb der Community der szenisch Forschenden und darauf basierend die Re- bzw. Neuformulierung von Kriterien und Ansprüchen notwendig sein. Fragen nach dem Grad, der Art und Weise der Partizipation der Mitwirkenden auch beim Verfassen von fachlichen Publikationen im Sinne vielstimmiger Dar- 
stellungsformen sowie die Weiterentwicklung und Anwendung ethischer Kriterien und Regeln werden dabei eine besondere Rolle spielen, wenn sich Forumtheater als szenischer und partizipativer Forschungsansatz in der sozialwissenschaftlichen und damit auch sozialpädagogischen Forschungslandschaft weiter etablieren will. Die spezifischen Beiträge und Leistungen von Forumtheater als szenischem Forschungsansatz in Handlungs- und Forschungsfeldern der Sozialen Arbeit bestehen meines Erachtens in der unmittelbaren Beteiligung der Adressat_innen, der Untersuchung von Handlungsspielräumen bei gleichzeitiger Bewusstheit der strukturellen Rahmungen sowie in der vielschichtigen, interaktiven Vermittlung von Erkenntnissen an gesellschaftliche und politische Öffentlichkeit(en). Dies entspricht einem von Schneider (2014) formulierten Verständnis von Forschung in der Sozialen Arbeit, das von ,der Sozialen Arbeit als Handlungswissenschaft in der Sorge um die soziale Existenz des Menschen ausgeh[t]" (Schneider 2014, S. 396). Dabei ist sie dem Handeln auf einer individuellen, institutionellen und politischen Ebene verpflichtet. Ebenso wichtig ist aber auch, dass sich Forschung stärker als bisher um Dissemination aktiv bemühen sollte. Denn eine Soziale Arbeit, so Schneider (2012, S. 165 f.), die gesellschaftlichen Wandel betreiben will, muss proaktiv und professionell agieren. Und dazu kann szenisches Forschen mit dem Forumtheater jedenfalls beitragen.

Funding Open access funding provided by University of Graz.

Open Access Dieser Artikel wird unter der Creative Commons Namensnennung 4.0 International Lizenz (http://creativecommons.org/licenses/by/4.0/deed.de) veröffentlicht, welche die Nutzung, Vervielfältigung, Bearbeitung, Verbreitung und Wiedergabe in jeglichem Medium und Format erlaubt, sofern Sie den/die ursprünglichen Autor(en) und die Quelle ordnungsgemäß nennen, einen Link zur Creative Commons Lizenz beifügen und angeben, ob Änderungen vorgenommen wurden.

\section{Literatur}

Anhorn, Roland, und Johannes Stehr. 2012. Grundmodelle von Gesellschaft und soziale Ausschließung: Zum Gegenstand einer kritischen Forschungsperspektive in der Sozialen Arbeit. In Kritisches Forschen in der Sozialen Arbeit. Gegenstandbereiche - Kontextbedingungen - Positionierungen - Perspektiven, Hrsg. Elke Schimpf, Johannes Stehr, 57-76. Wiesbaden: VS.

Bergold, Jarg, und Stefan Thomas. 2012. Partizipative Forschungsmethoden: Ein methodischer Ansatz in Bewegung. Forum Qualitative Sozialforschung https://doi.org/10.17169/fqs-13.1.1801.

Boal, Augusto. 1999. Der Regenbogen der Wünsche: Methoden aus Theater und Therapie. Seelze (Velber): Kallmeyer.

Boal, Augusto. 2013. Übungen und Spiele für Schauspieler und Nicht-Schauspieler. Aktualisierte und erweiterte Ausgabe. Frankfurt a. Main: Suhrkamp. Herausgegeben und aus dem brasilianischen Portugiesisch übersetzt von Till Baumann.

Bülow-Schramm, Margret, und Dietlinde Gipser (Hrsg.). 1997. Spielort Universität. 10 Jahre Lehr-/ Lernprojekt „Der brüchige Habitus“. Hochschuldidaktische Arbeitspapiere, Bd. 30. Hamburg: Interdisziplinäres Zentrum für Hochschuldidaktik der Universität Hamburg.

Bülow-Schramm, Margret, und Dietlinde Gipser. 2007. Theater mit dem Habitus. Zur forschungsbasierten szenischen Arbeit am brüchigen Habitus von HochschullehrerInnen. In Bühne frei für Forschungstheater. Theatrale Inszenierungen als wissenschaftlicher Erkenntnisprozess, Hrsg. Margret BülowSchramm, Dietlinde Gipser, und Doris Krohn, 87-104. Oldenburg: Paolo Freire.

Diamond, David. 2013. Theater zum Leben. Über die Kunst und die Wissenschaft des Dialogs in Gemeinwesen. Stuttgart: Ibidem.

Fritz, Birgit. 2011. InExActArt. Ein Handbuch zur Praxis des Theaters der Unterdrückten. Stuttgart: Ibidem. 
Fritz, Birgit. 2013. Von Revolution zu Autopoiese. Auf den Spuren Augusto Boals ins 21. Jahrhundert. Das Theater der Unterdrückten im Kontext von Friedensarbeit und einer Ästhetik der Wahrnehmung. Stuttgart: Ibidem.

Garfinkel, Harold. 1985. Studies in Ethnomethodology. Oxford: Polity Press.

Gipser, Dietlinde. 1996. Grenzüberschreitungen: Theater der Unterdrückten an Hochschulen in Nah-Ost und West - Emanzipatorische Forschungsprozesse. zeitschrift für befreiende pädagogik 10:26-31.

Gipser, Dietlinde. 2007. Hochschultheater. Wege zu einer emanzipatorischen Forschungspraxis. In Bühne frei für Forschungstheater. Theatrale Inszenierungen als wissenschaftlicher Erkenntnisprozess, Hrsg. Margret Bülow-Schramm, Dietlinde Gipser, und Doris Krohn, 127-160. Oldenburg: Paolo Freire.

Graßhoff, Gunther (Hrsg.). 2013. Adressaten, Nutzer, Agency. Akteursbezogene Forschungsperspektiven in der Sozialen Arbeit. Wiesbaden: VS.

Guhathakurta, Meghna. 2015. Theatre in participatory action research: Experiences from Bangladesh. In The SAGE Handbook of Action Research, Hrsg. Hilary Bradbury, 100-108. Los Angeles: SAGE.

Hanses, Andreas. 2005. AdressatInnenforschung in der Sozialen Arbeit - Zwischen disziplinärer Grundlegung und Provokation. In Sozialpädagogik als forschende Disziplin, Hrsg. Cornelia Schweppe, Werner Thole, 185-199. Weinheim, München: Juventa.

Koch, Gerd. 1988. Lernen mit Bert Brecht. Bertolt Brechts politisch-kulturelle Pädagogik. Frankfurt a. Main: Brandes \& Apsel.

Koch, Gerd. 1997. Theater-Spiel als szenische Sozialforschung. In TheaterSpiel. Ästhetik des Schul- und Amateurtheaters, Hrsg. Jürgen Belgrad, 81-96. Hohengehren: Schneider.

Kramer, Paula. 2007. Performing Research-Researching Performance in South Africa. Methodological Musings on Research Praxis and Theatre as Research. In Bühne frei für Forschungstheater. Theatrale Inszenierungen als wissenschaftlicher Erkenntnisprozess, Hrsg. Margret Bülow-Schramm, Dietlinde Gipser, und Doris Krohn, 171-182. Oldenburg: Paolo Freire.

Liamputtong, Pranee. 2007. Researching the vulnerable. A guide to sensitive research methods. London, Thousand Oaks, New Dehli: SAGE.

Linds, Warren, und Elinor Vettraino. 2008. Collective imagining: Collaborative story telling through image theater [54 paragraphs]. Forum Qualitative Sozialforschung/Forum: Qualitative Social Research 9(2):56.

Milgram, Stanley. 1974. Obedience to authority. An experimental view. New York: Harper.

Schimpf, Elke. 2012. Widersprüchliche Deutungsmuster und Praktiken lebensweltorientierten Forschens. In Kritisches Forschen in der Sozialen Arbeit. Gegenstandbereiche - Kontextbedingungen - Positionierungen - Perspektiven, Hrsg. Elke Schimpf, Johannes Stehr, 233-261. Wiesbaden: VS.

Schneider, Armin. 2012. Forschung und Soziale Arbeit - Chancen und Grenzen zur Politikbeeinflussung. In Forschung, Politik und Soziale Arbeit, Hrsg. Armin Schneider, Rebekka Streck, und Eppler Natalie, 163-177. Opladen, Berlin, Toronto: Budrich.

Schneider, Armin. 2014. Forschung. In Handlungsräume Sozialer Arbeit, Hrsg. Günter J. Friesenhain, Daniela Braun, und Rainer Ningel, 392-400. Opladen, Toronto: Barbara Budrich.

Seitz, Hanne. 2012. Performative Research. In Die Kunst, über kulturelle Bildung zu forschen. Theorie- und Forschungsansätze, Hrsg. Tobias Fink, Burkhard Hill, Vanessa-Isabelle Reinwand, und Alexander Wenzlik, 81-95. München: kopaed.

Staffler, Armin. 2009. Augusto Boal. Einführung. Essen: Oldib.

Streck, Danilo R., und Oscar Jara Holliday. 2015. Research, participation and social transformation: Grounding systematization of experiences in Latin American perspectives. In The SAGE Handbook of Action Research, Hrsg. Hilary Bradbury, 472-480. Los Angeles: SAGE.

von Unger, Hella. 2014. Partizipative Forschung. Einführung in die Forschungspraxis. Wiesbaden: VS.

Wakefort, Tom, Michael Pimbert, und Eric Walcon. 2015. Re-fashioning citizens' juries: Participatory democracy in action. In The SAGE Handbook of Action Research, Hrsg. Hilary Bradbury, 230-246. Los Angeles: SAGE.

Weitzel, Julia. 2012a. Existenzielle Bildung. Zur ästhetischen und szenologischen Aktualisierung einer bildungstheoretischen Leitidee. Bielefeld: transcript.

Weitzel, Julia. 2012b. Forschungslabor: Szenisches Forschen. In Die Kunst, über kulturelle Bildung zu forschen. Theorie- und Forschungsansätze, Hrsg. Tobias Fink, Burkhard Hill, Vanessa-Isabelle Reinwand, und Alexander Wenzlik, 262-271. München: kopaed.

Winter, Rainer. 2010. Ein Plädoyer für kritische Perspektiven in der qualitativen Forschung [41 Absätze]. Forum Qualitative Sozialforschung/Forum: Qualitative Social Research 12(1):7

Winter, Rainer. 2012. Macht, Kultur und soziale Intervention. Cultural Studies als kritische Theorieperspektive in der Sozialen Arbeit. In Kritik der Sozialen Arbeit - Kritische Soziale Arbeit, Hrsg. Roland Anhorn, Frank Bettinger, Cornelis Horlacher, und Kerstin Rathgeb, 449-462. Wiesbaden: VS. 
Wrentschur, Michael. 2004. Theaterpädagogische Wege in den öffentlichen Raum. Zwischen struktureller Gewalt und lebendiger Beteiligung. Stuttgart: Ibidem.

Wrentschur, Michael. 2007. Forschen mit Methoden des Theaters und des szenischen Spiels. Ein Zwischenbericht. In Bühne frei für Forschungstheater. Theatrale Inszenierungen als wissenschaftlicher Erkenntnisprozess, Hrsg. Margret Bülow-Schramm, Dietlinde Gipser, und Doris Krohn, 127-160. Oldenburg: Paolo Freire.

Wrentschur, Michael. 2008a. Forum theatre as a participatory tool for social research and development: a reflection on nobody is perfect: a project with homeless people. In Qualitative research and social change in European contexts, Hrsg. Pat Cox, Thomas Geisen, und Roger Green, 94-111. New York: palgrave macmillan.

Wrentschur, Michael. 2008b. Kein Kies zum Kurven Kratzen - Neuer Armut entgegenwirken. Ein Projekt zur kreativen Beteiligung von Armutsbetroffenen. In Armut, Gesellschaft und Soziale Arbeit. Perspektiven gegen Armut und soziale Ausgrenzung in Österreich, Hrsg. Gerald Knapp, Heinz Pichler, 692-723. Klagenfurt, Ljubljana, Wien: Hermagoras.

Wrentschur, Michael. 2010. Neuer Armut entgegenwirken: Politisch-partizipative Theaterarbeit als kreativer Impulse für soziale und politische Partizipationsprozesse. In Macht - Eigensinn - Engagement. Lernprozesse gesellschaftlicher Teilhabe, Hrsg. Angela Pilch Ortega, Andrea Felbinger, Regina Mikula, und Rudolf Egger, 211-232. Wiesbaden: VS.

Wrentschur, Michael. 2011. Theater an die Macht: Neuer Armut entgegenwirken - bis ins Parlament! Zeitschrift für Theaterpädagogik, Korrespondenzen 27(58):55-58.

Wrentschur, Michael. 2012. Szenisch-partizipatives Forschen als Beitrag zu sozialer Teilhabe und politischer Beteiligung. In Empirische Forschung in der Sozialen Arbeit. Methoden und methodologische Herausforderungen, Hrsg. Arno Heimgartner, Ulrike Loch, und Stephan Sting, 137-153. Wien, Berlin, Münster: LIT.

Wrentschur, Michael. 2014. Die Stimmen der AdressatInnen - werden sie gehört? Situative Blitzlichter auf das Zusammenspiel von Anerkennung, Respekt und Partizipation. In Perspektiven der AkteurInnen in der Sozialen Arbeit, Hrsg. Arno Heimgartner, Karin Lauermann, und Stephan Sting, 69-82. Wien, Berlin, Münster: LIT.

Wrentschur, Michael. 2019. Forumtheater, szenisches Forschen und Soziale Arbeit. Diskurse - Verfahren - Fallstudien. Weinheim, Basel: Beltz Juventa.

Wrentschur, Michael, und Michaela Moser. 2014. ,Stop: Now we are speaking!‘ A creative and dissident approach of empowering disadvantaged young people. International Social Work 57(4):398-410.

Michael Wrentschur PD Mag. Dr., lehrt und forscht am Institut für Erziehungs- und Bildungswissenschaft der Karl-Franzens-Universität Graz im Arbeitsbereich Sozialpädagogik. Schwerpunkte: Theaterarbeit in sozialen Feldern, Soziokultur und soziale Kulturarbeit, Armut und soziale Ausgrenzung, Partizipation; szenische und partizipative Forschungsmethoden; Künstlerischer Leiter von InterACT, der Werkstatt für Theater und Soziokultur. michael.wrentschur@uni-graz.atwww.interact-online.org 